\section{Case Reports in Ophthalmology}

\title{
Corneal Phaeohyphomycosis Caused by Bipolaris hawaiiensis
}

\author{
Winai Chaidaroon ${ }^{a} \quad$ Sumet Supalaset $^{\mathrm{a}}$ Napaporn Tananuvat $^{\mathrm{a}}$ \\ Nongnuch Vanittanakom ${ }^{b}$ \\ ${ }^{a}$ Department of Ophthalmology, Faculty of Medicine, Chiang Mai University, Chiang \\ Mai, Thailand; 'Department of Microbiology, Faculty of Medicine, Chiang Mai University, \\ Chiang Mai, Thailand
}

\section{Keywords}

Bipolaris hawaiiensis - Phaeohyphomycosis - Keratitis - Dematiaceous fungus

\begin{abstract}
Purpose: To report a rare case of keratitis infected by Bipolaris hawaiiensis. Methods: A patient who was diagnosed as fungal keratitis caused by $B$. hawaïensis was retrospectively reviewed for history, clinical characteristics, risk factors, laboratory findings, treatments, and outcomes. Results: A 63-year-old man with a history of trauma and saw dust in the left eye presented with a corneal ulcer. Eye examination revealed whitish infiltration with a feathery edge and small brownish deposits in the anterior stroma of the left cornea. Numerous septate hyphal fragments were detected in a corneal specimen, and nucleotide sequence analysis identified B. hawaiiensis. Treatment was started with 5\% natamycin eyedrops and oral itraconazole. Subsequently, a corneal plaque developed which did not respond to medication and debridement. The patient underwent therapeutic penetrating keratoplasty. Conclusions: $B$. hawaiiensis is a rare cause of corneal phaeohyphomycosis. A brownish pigmented infiltration is an important diagnostic clue, however microbiologic studies are required to obtain a definite diagnosis. Although antifungal medication and debridement are the mainstay of most corneal fungal infection, therapeutic penetrating keratoplasty can prevent morbidity related to this fungal infection.




\section{Case Reports in Ophthalmology}

\section{Introduction}

Fungal keratitis is a common cause of keratitis in tropical and semitropical countries [1]. Most of these infections are caused by filamentous fungi. Aspergillus and Fusarium species are the etiology of the majority of keratomycoses in many reports worldwide [2-5]. Dematiaceous fungi have increasingly obtained importance as organisms that cause corneal ulcer [6]. Species of Curvularia and Bipolaris, which belong to dematiaceous fungi, are the third most important cause of keratomycosis in many studies [6-8].

Bipolaris hawaiiensis is a darkly pigmented fungus that can cause subcutaneous, cutaneous and soft tissue diseases known as phaeohyphomycosis [9]. This organism is widely distributed in nature and most frequently related to plant material, grasses and soil. It is known to cause bronchopulmonary disease, encephalitis and subcutaneous phaeohyphomycosis [10-12], although there are a limited number of reports of ocular infection caused by $B$. hawaiiensis $[13,14]$. B. hawaiiensis is an uncommon cause of keratitis. We report a refractory case of $B$. hawaiiensis keratitis, presenting after eye trauma, including clinical features, management, and treatment outcome.

\section{Case History}

A 63-year-old male patient reported a history of trauma to the left eye with sawdust while sawing wood 5 months earlier. The patient had underlying ocular hypertension in his right eye, but no other predisposing factors such as diabetes, topical steroid use, or immunocompromised state. He went to a secondary hospital where he was diagnosed with corneal ulcer. He was treated for fungal keratitis with $0.15 \%$ amphotericin B in the left eye and ocular hypertension in the right eye for 5 months as an outpatient. The lesion did not improve. Thus, the patient was referred to the ophthalmological outpatient department of Chiang Mai University Hospital in July 2015.

The patient complained of decreased vision and of photophobia in the left eye. Best corrected visual acuity (BCVA) was 6/6 in the right eye and 6/12 in the left eye. Eye examination revealed a whitish infiltration with a feathery edge and a brownish pigmented lesion in the anterior stroma of left central cornea. Corneal thinning, epithelial defect, mild anterior chamber inflammation, and moderate conjunctival injection were observed (fig. 1a).

After admission to the hospital, $0.15 \%$ amphotericin B was discontinued. The corneal lesion was scraped for potassium hydroxide $(\mathrm{KOH})$ examination, Gram staining, calcofluorwhite staining, and culture. The Gram staining showed few septate hyphae. The KOH examination and calcofluor-white staining showed numerous septate hyphae (fig. $2 \mathrm{a}$, b). Confocal microscopy was performed on the left eye and demonstrated acute angle branching hyphae (fig. 2c). Fasting blood sugar was normal, and HIV antibody test was negative.

Treatment was started with 5\% natamycin eyedrops hourly, $1 \%$ atropine four times per day, and $0.18 \%$ sodium hyaluronate every $4 \mathrm{~h}$ to the left eye. The right eye was treated with $0.5 \%$ timolol twice per day, brimonidine three times per day, and bimatoprost at night for ocular hypertension. Itraconazole $(100 \mathrm{mg}) 2$ tablets daily, doxycycline $(100 \mathrm{mg}) 1$ tablet twice per day, and vitamin C (500 mg) 1 tablet twice per day were prescribed for 1 week. Liver function was monitored routinely throughout the course of the treatment. The edge of the ulcer was well demarcated, indicating gradual improvement of the eye lesion. Therefore, the $5 \%$ natamycin eyedrops treatment was decreased to a frequency of every $2 \mathrm{~h}$ for the next 3 weeks. 


\section{Case Reports in Ophthalmology}

The central lesion remained densely infiltrated, with an overlying superficial corneal plaque and epithelial defect (fig. 1b). Corneal debridement was done in order to remove all plaques, and the specimen was sent for $\mathrm{KOH}$ examination, calcofluor-white staining and culture once again. The result showed numerous septate hyphae. The result of the previous fungal culture revealed numerous dematiaceous septate hyphae of Bipolaris spp. (fig. 2d).

At week 5 after admission, the lesion seemed to be unresponsive to natamycin. Hence, subconjunctival injection of fluconazole $(0.5 \mathrm{ml})$ was started on alternate days and $5 \%$ natamycin eyedrops was reduced to every $4 \mathrm{~h}$ for 2 weeks. All other medications remained unchanged.

Therapeutic corneal debridement was performed. Unfortunately, the lesion did not improve. The dense infiltrate remained, with overlying recalcitrant superficial corneal plaque. There was also an epithelial defect at the central cornea (fig. 1c). BCVA was 6/6 in the right eye and hand movement in the left eye. The corneal ulcer seemed refractory to medications. Therefore, therapeutic penetrating keratoplasty was performed (fig. 1d). A corneal specimen was sent for pathologic investigation. Special staining with Gomori methenamine silver, periodic acid-Schiff, and hematoxylin \& eosin revealed septate fungal hyphae with acute angle branching (fig. 3).

On postoperative day 1 , BCVA was 6/6 in the right eye and 6/60 in the left eye. Topical and systemic antifungal therapy was continued in the postoperative period. Treatment consisted of $5 \%$ natamycin eyedrops every $4 \mathrm{~h}, 0.5 \%$ levofloxacin ophthalmic solution four times per day, and 1\% atropine four times per day in the left eye. Itraconazole (100 mg) 2 tablets daily was given orally for 2 weeks. Then, $1 \%$ prednisolone acetate ophthalmic suspension was started every $4 \mathrm{~h}$ once the infection was controlled. Within 2 weeks postoperatively, the corneal graft was clear, with no recurrence of infiltration. There were no early postoperative complications. The result of nucleotide sequence analysis identified the organism as B. hawaiiensis. BCVA had improved to $6 / 24$ in the left eye 3 months after the operation. The corneal graft remained clear without recurrent infection.

\section{Discussion}

In humans, Bipolaris is classified as a dematiaceous or darkly pigmented fungus that causes phaeohyphomycosis [9]. It rarely infects humans. The most frequently reported species are B. spicifera, B. australiensis, and B. hawaiiensis [15]. Previous studies have shown that $B$. hawaiiensis can infect a variety of organs [10-12]. The case reports of corneal ulcer caused by $B$. hawaiiensis are few because $B$. hawaiiensis is an uncommon cause of keratitis. One has been reported in a leprosy patient by Anandi et al. [14] and another in an immunocompetent patient by Bashir et al. [13].

This case of B. hawaiiensis keratomycosis was found in Thailand, a tropical country like India, in both of which $B$. hawaiiensis keratomycosis has been reported previously $[13,14]$. The predisposing factor of this case, a male with outdoor trauma, is in accordance with Garg et al.'s [6] report of the most common predisposing factor for dematiaceous fungal keratitis. It is possible that $B$. hawaiiensis is widely distributed in plants or soil. Therefore, it could infect humans by contact with contaminated plant debris, wood or soil. The clinical features of this case, namely the whitish infiltrate with a feathery edge in the anterior stroma of the central cornea and brownish pigmented lesion, look no different from those reported by Garg et al. [6], who found yellow-white infiltrate with dry, raised slough and feathery edges in most cases of dematiaceous fungal keratitis. However, this case developed a whitish cor- 
Case Reports in
Ophthalmology

Case Rep Ophthalmol 2016;7:364-371 $10.1159 / 000447737$

C 2016 The Author(s). Published by S. Karger AG, Basel www.karger.com/cop

Chaidaroon et al.: Corneal Phaeohyphomycosis Caused by Bipolaris hawaiiensis

neal plaque as the specific characteristic, which was previously reported with rare organisms $[15,16]$. The recalcitrant corneal plaques may prevent the penetration of drugs. On the other hand, corneal plaques seemed to simultaneously strengthen the corneal stroma and prevent perforation of the cornea. In addition, the previous misdiagnosis as yeast and inappropriate treatment caused a delay in appropriate treatment. $\mathrm{KOH}$ examination and calcofluor-white staining revealed numerous septate hyphae. Fungal culture showed dark septate hyphae with numerous conidia of Bipolaris spp. Nucleotide sequence analysis confirmed the diagnosis of $B$. hawaiiensis.

The authors encourage clinicians to perform more specific mycological evaluations of these pigmented fungi to better define their roles in fungal keratitis. Thorough evaluation and accurate diagnosis will lead to the appropriate treatment. In this case, topical natamycin was initially started. The manifestation of the whitish corneal plaque complicated the treatment. Although use of topical natamycin has been reported to be successful in treating most dematiaceous fungal keratitis [6], this case did not respond to topical natamycin and corneal debridement. Beside natamycin, nystatin ointment [14] is another treatment for $B$. hawaiiensis. Unfortunately nystatin ointment is not available in Thailand. Subconjunctival fluconazole injection [15] was attempted, but unfortunately the lesion did not improve. Eventually, therapeutic penetrating keratoplasty was required.

Considering penetrating keratoplasty in infectious keratitis, the advantages include the ability to remove infected and inflamed corneal tissue unresponsive to medical treatment, shortened hospitalization and rapid improvement in visual acuity. However, the disadvantages that need to be addressed are the risk of corneal graft failure, recurrent infectious keratitis in case of total involvement of corneal ulcer, the high incidence of graft failure, and the risk of glaucoma due to topical steroid use [17].

In this study, we report the success of therapeutic penetrating keratoplasty in treating B. hawaiiensis keratomycosis which did not respond to medications, and conclude that therapeutic penetrating keratoplasty should be considered to remove the infected corneal tissue and recalcitrant corneal plaque and to improve visual acuity in refractory B. hawaiiensis keratomycosis.

\section{Conclusion}

B. hawaiiensis is a virulent organism which can cause ocular morbidity and blindness. Early diagnosis and accurate identification of the pathogenic B. hawaiiensis can lead to prompt and appropriate treatment and prevent ocular morbidity and blindness. In refractory $B$. hawaiiensis keratomycosis, therapeutic penetrating keratoplasty should be considered to remove the infected corneal tissue and recalcitrant corneal plaque and to improve visual acuity.

\section{Acknowledgment}

The authors gratefully acknowledge Dr. Pongsak Mahanupap, MD, for providing photographs of the histologic stainings. 


\section{Statement of Ethics}

The authors have no ethical conflicts to disclose.

\section{Disclosure Statement}

None of the authors has any conflicts of interest concerning this case report.

\section{References}

1 Chander J, Singla N, Agnihotri N, Arya SK, Deep A: Keratomycosis in and around Chandigarh: a five-year study from a north Indian tertiary care hospital. Indian J Pathol Microbiol 2008;51:304-306.

Chowdhary A, Singh K: Spectrum of fungal keratitis in North India. Cornea 2005;24:8-15.

-3 Tanure MA, Cohen EJ, Sudesh S, Rapuano CJ, Laibson PR: Spectrum of fungal keratitis at Wills Eye Hospital, Philadelphia, Pennsylvania. Cornea 2000;19:307-312.

4 Panda A, Sharma N, Das G, Kumar N, Satpathy G: Mycotic keratitis in children: epidemiological and microbiological evaluation. Cornea 1997;16:295-299.

5 Chander J, Sharma A: Prevalence of fungal corneal ulcer in North India. Infection 1994;22:207-209.

-6 Garg P, Gopinathan U, Choudhary K, Rao GN: Keratomycosis: clinical and microbiologic experience with dematiaceous fungi. Ophthalmology 2000;107:574-580.

7 Gopinathan U, Garg P, Fernandes M, Sharma S, Athmanathan S, Rao GN: The epidemiological features and laboratory results of fungal keratitis: a 10 -year review at a referral eye care center in South India. Cornea 2002;21:555-559.

8 Leck AK, Thomas PA, Hagan M, Kaliamurthy J, Ackuaku E, John M, Newman MJ, Codjoe FS, Opintan JA, Kalavathy CM, Essuman V, Jesudasan CA, Johnson GJ: Aetiology of suppurative corneal ulcers in Ghana and South India, and epidemiology of fungal keratitis. Br J Ophthalmol 2002;86:1211-1215.

-9 Ajello L, Georg LK, Steigbigel RT, Wang CJ: A case of phaeohyphomycosis caused by a new species of Phialophora. Mycologia 1974;66:490-498.

10 Saenz RE, Brown WD, Sanders CV: Allergic bronchopulmonary disease caused by Bipolaris hawaiiensis presenting as a necrotizing pneumonia: case report and review of literature. Am J Med Sci 2001;321: 209-212.

11 Morton SJ, Midthun K, Merz WG: Granulomatous encephalitis caused by Bipolaris hawaiiensis. Arch Pathol Lab Med 1986;110:1183-1185.

12 Verma R, Roy P, Vasudevan B, Bhatt P, Kharayat V, Kaur G: Subcutaneous phaeohyphomycosis caused by Bipolaris hawaiiensis in an immunocompetent patient. Indian J Dermatol Venereol Leprol 2014;80: 554-556.

13 Bashir G, Hussain W, Rizvi A: Bipolaris hawaiiensis keratomycosis and endophthalmitis. Mycopathologia 2009;167:51-53.

14 Anandi V, Suryawanshi NB, Koshi G, Padhye AA, Ajello L: Corneal ulcer caused by Bipolaris hawaiiensis. J Med Vet Mycol 1988;26:301-306.

15 da Cunha KC, Sutton DA, Fothergill AW, Cano J, Gené J, Madrid H, De Hoog S, Crous PW, Guarro J: Diversity of Bipolaris species in clinical samples in the United States and their antifungal susceptibility profiles. J Clin Microbiol 2012;50:4061-4066.

16 Cho BJ, Lee YB: Infectious keratitis manifesting as a white plaque on the cornea. Arch Ophthalmol 2002;120:1091-1093.

-17 Chaidaroon W, Ausayakhun S, Ngamtiphakorn S, Prasitsilp J: Clinical indications for penetrating keratoplasty in Maharaj Nakorn Chiang Mai Hospital, 1996-1999. J Med Assoc Thai 2003;86:206-211. 

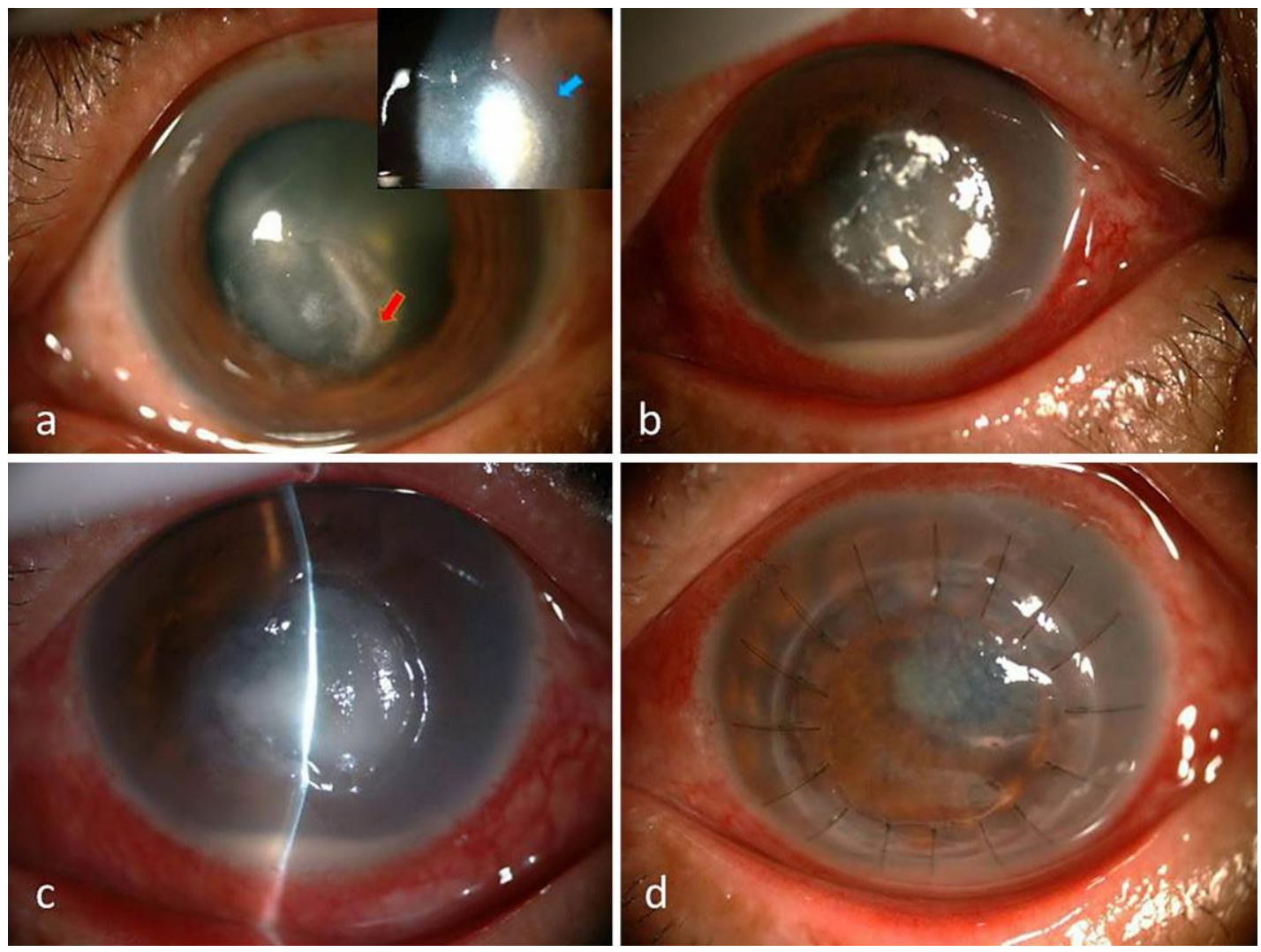

Fig. 1. Photographs of the left eye before and after treatment. a At first visit, the whitish infiltration with a macroscopic feathery edge (blue arrow) and the macroscopic pigmentation (red arrow) were located in anterior stroma of cornea. b After 3 weeks of medical treatment, the overlying superficial corneal white plaque had developed. c After 5 weeks of medical treatment, the hypopyon and the epithelial defect had developed. $\mathbf{d}$ One day after therapeutic penetrating keratoplasty, the corneal graft was clear. 


\section{Case Reports in Ophthalmology}
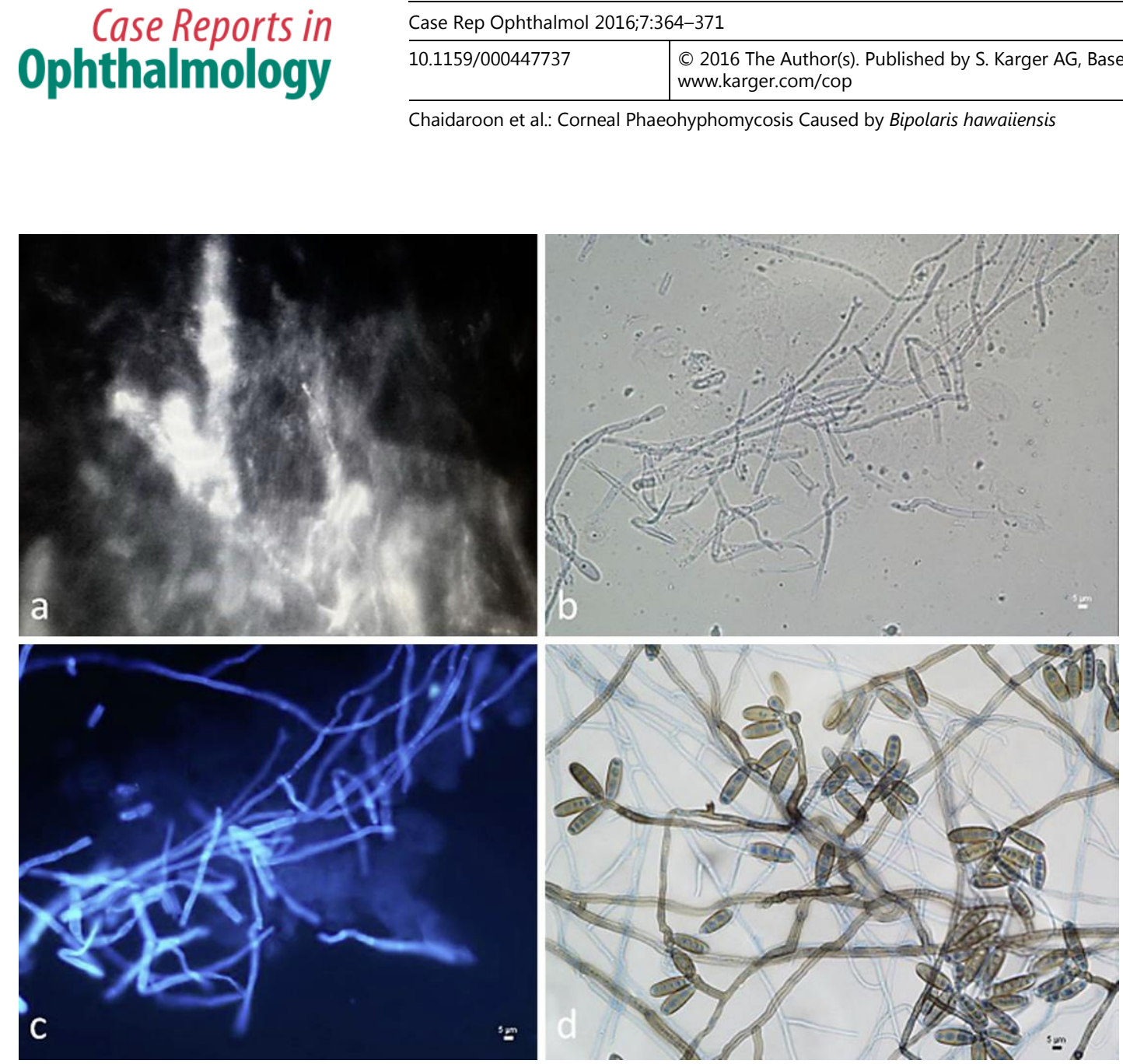

Fig. 2. a Confocal microscopy demonstrated acute angle branching hyphae. b KOH preparation showed numerous septate hyphae. c Calcofluor-white staining also showed numerous septate hyphae. d Fungal culture revealed numerous dematiaceous septate hyphae of Bipolaris spp. Magnification: $\times 400$. 


\section{Case Reports in Case Rep Ophthalmol 2016;7:364-371 \\ C 2016 The Author(s). Published by S. Karger AG, Basel www.karger.com/cop \\ Chaidaroon et al.: Corneal Phaeohyphomycosis Caused by Bipolaris hawaiiensis}
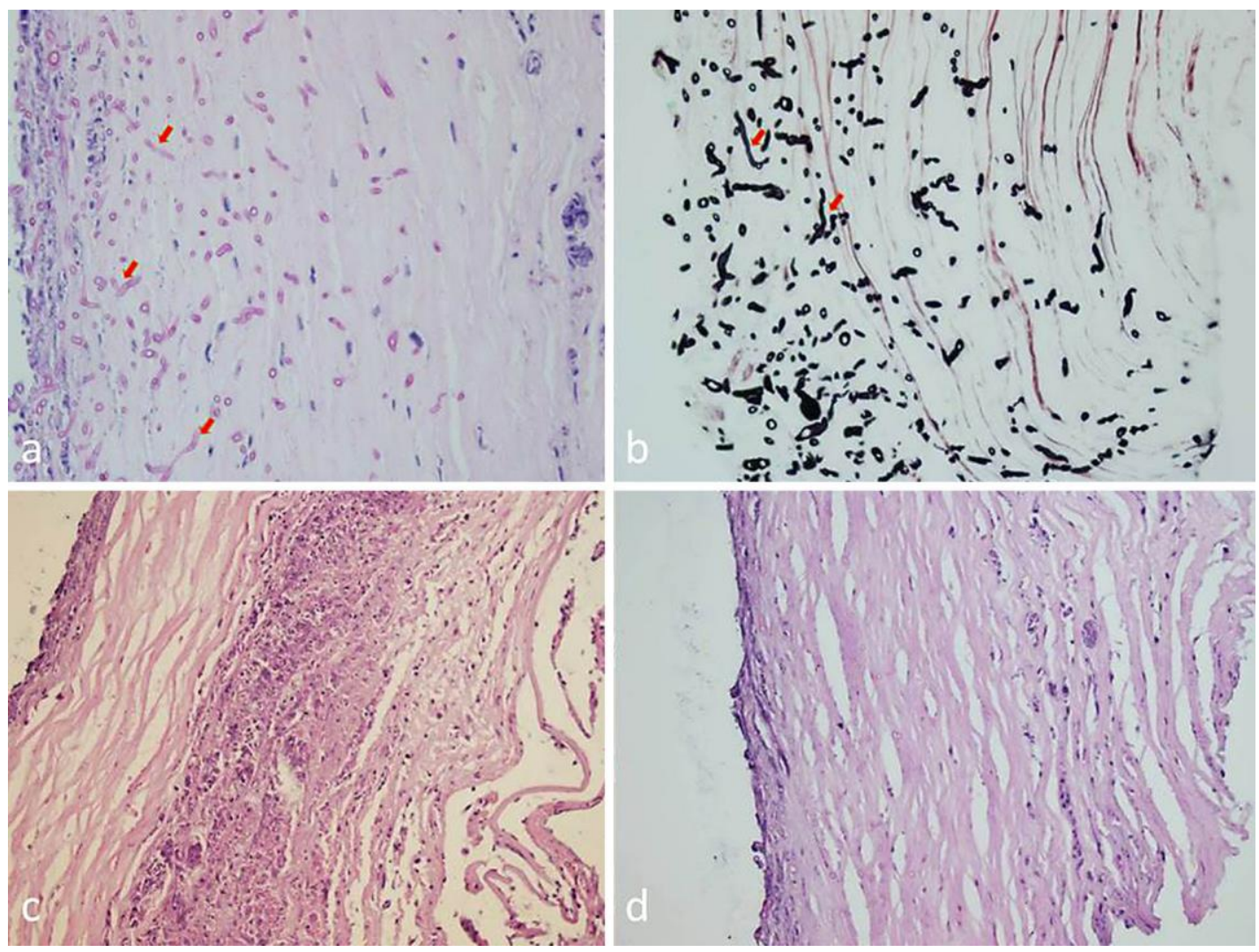

Fig. 3. Special stains revealed numerous septate fungal hyphae in the corneal stroma. a Periodic acid-Schiff. Magnification: $\times 400$. b Gomori methenamine silver. Magnification: $\times 400$. c, $\mathbf{d}$ Hematoxylin \& eosin. Magnification: $\times 400(c)$ and $\times 600(d)$. 